Modeling, Identification and Control, Vol. 39, No. 2, 2018, pp. 61-72, ISSN 1890-1328

\title{
Lifetime Calculation of Irregularly Oscillating Bearings in Offshore Winches
}

\author{
L. Wöll G. Jacobs A. Kramer \\ RWTH Aachen University, Institute for Machine Elements and Systems Engineering, Schinkelstr. 10, DE-52062 \\ Aachen, Germany. E-mail: \{lothar.woell,georg.jacobs,achim.kramer\} @imse.rwth-aachen.de
}

\begin{abstract}
The fatigue lifetime calculation of rolling bearings has been intensively researched in the past and has been well documented in standards. The majority of the conducted research in the field of bearing life expectancy only applies to bearings under continuously rotating conditions as can be found in many technical systems, such as vehicle transmissions. However, there are many other technical applications in which bearings perform oscillating motions, for which the fatigue life calculation is currently not standardized. For such oscillatory behaviour, there exist several modifications for the standardized lifetime calculation. The available modification approaches, however, assume purely sinusoidal oscillations. Thus, irregular non-sinusoidal oscillations require approximation with a simple sinusoidal oscillation to comply with the available approaches, which can lead to a loss of information.

In this paper, a numerical calculation approach to modify the lifetime calculation for oscillating bearings underlying irregularly reversing speeds and varying loads is presented. The lifetime calculation results of this approach are compared to results of existing calculation approaches for fatigue life calculation of oscillating bearings. For this, the approaches are applied on simple sinusoidal speeds and loads. Subsequently, results of all calculation approaches are shown for irregularly varying speeds and loads, using the example of the drivetrain of an offshore crane winch with Active Heave Compensation (AHC). The calculation results indicate, that available lifetime modification approaches for oscillating bearings seem to overestimate the lifetime for irregularly oscillating conditions.
\end{abstract}

Keywords: Oscillation, Lifetime, Reliability, Offshore, Gearbox, Winch, Active Heave Compensation

\section{Introduction}

Fatigue lifetime calculations of rolling bearings are very well researched and documented in standards such as ISO 281 (2007). The provided lifetime calculation approaches are designed to calculate the lifetime of rolling bearings under continuously rotating conditions, as can be found in numerous mechanical systems, e.g. vehicle transmissions or wind turbine drivetrains. However, there are many applications in which bearings perform only oscillating motions. For such cases, e.g. wind turbine pitch drives or drivetrains of offshore crane winches with active heave compensation, the bearing lifetime calculation is not simply assessable using the documented approach in the standard ISO 281. There are several approaches available to calculate modification factors for adaptation of the basic fatigue lifetime calculation of continuously rotating bearings to bearings with oscillating behaviour. For oscillations with very small angles, damage mechanisms such as False Brinelling and Fretting Corrosion can occur. Since only lifetime calculation due to fatigue is thoroughly researched and documented, restrictions regarding damage mechanisms must be made to apply any modifications to the standard ISO 281 (Harris et al., 2009).

The available modification approaches are designed 
to analyse purely sinusoidal oscillations. For many applications, bearings are subject to irregular, nonsinusoidal motion, which require approximations with a simple sine to comply with the available approaches, see example in figure 1.

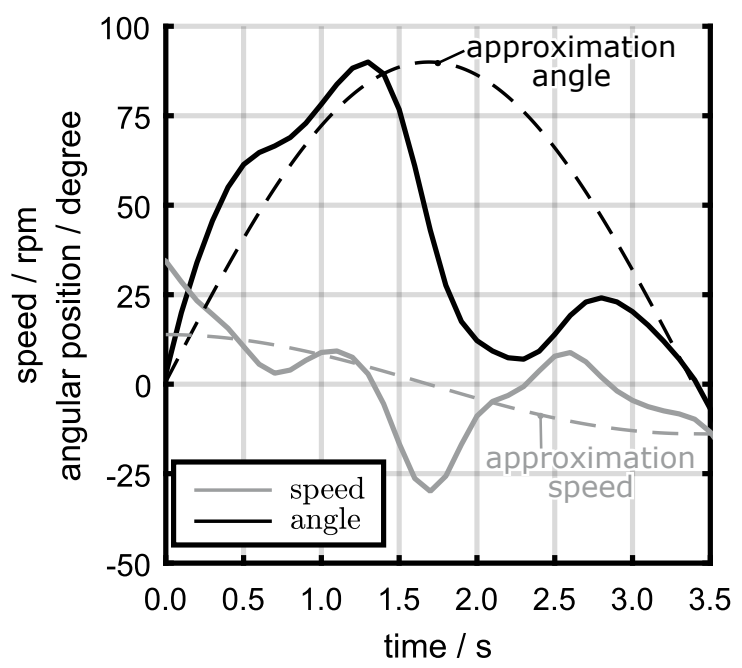

Figure 1: Exemplary irregular oscillation, approximated with simple sine

The sine approximating the angular motion has the same amplitude as the underlying irregular halfoscillation. The approximation of the speed is derived from the sinusoidal angular motion. Evidently, the underlying motion and the approximated motion differ from each other significantly, presumably leading to different lifetime results, which the available approaches are not able to assess.

Furthermore, the available methods only modify the basic lifetime calculation, but not the influence on the life modification factor $a_{I S O}$, described by ISO 281 , taking into account the non-linear influence of speed and load, among others, on the lubrication condition. Therefore, in this paper, an approach is presented for modifying the lifetime calculation for oscillating bearings underlying irregularly reversing speeds and varying loads to include the influence on the basic lifetime calculation as well as the life modification factor $a_{I S O}$.

This paper is structured as follows. The available approaches for lifetime calculation of oscillating bearings will be presented and discussed in section 2. Since these available approaches are designed only for sinusoidal oscillations, a numerical approach to consider irregular oscillations is presented in section 3. Thereafter, a comparison between the available approaches and the presented numerical approach is performed by applying the different approaches to an artificial bearing and sinusoidal duty cycle and calculating the re- spective lifetime modification factors. Furthermore, to demonstrate the influence of non-sinusoidal oscillations on the lifetime calculation results, all presented approaches will be applied to the bearings of an offshore crane winch drivetrain. The results will be discussed and an outlook will be given.

\section{State of the Art}

The lifetime of rolling bearings, limited by surfaceinitiated rolling fatigue, has been initially described by Lundberg and Palmgren (1947) and serves as a basis for the basic lifetime equation (1), provided by ISO 281 (2007).

$$
L_{10}=\left(\frac{C}{P}\right)^{p} \cdot 10^{6} \text { revolutions }
$$

The equation gives the relation between the dynamic equivalent load $P$, the bearing capacity $C$, the life exponent $p$ and the basic life rating $L_{10}$, corresponding to a failure probability of $10 \%$ (survival probability of $90 \%)$. The exponent $p$ depends on the kind of contact between rolling element and race. For point contacts, as can be found in ball bearings, a value of 3 is assumed. For ideal line contacts the value of $p$ is 4 . However, since the rolling elements in roller bearings are often profiled to achieve a better stress distribution and therefore the contact transforms from a point contact to a line contact for a certain load. Thus, an exponent $p$ with a value of $10 / 3$ for such roller bearings is suggested for the life rating calculation (Harris and Kotzalas, 2007).

The resulting life rating $L_{10}$ for continuously rotating conditions is expressed as number of full revolutions.

For oscillating motion with an amplitude $\phi$ around the centre position, however, the direction of rotation is periodically changing, see figure 2 .

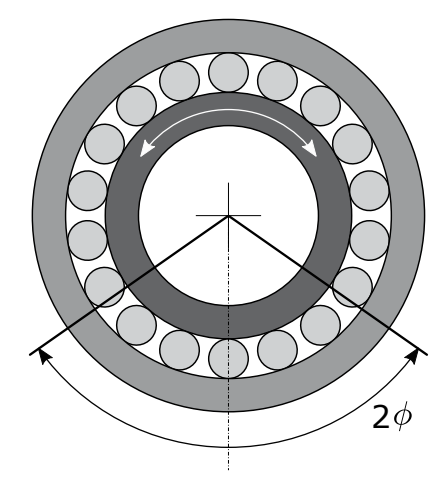

Figure 2: Oscillating Bearing 
To account for such motion, there exist several modifications to convert the speed and load data to comply with the ISO 281 lifetime calculation. These approaches have in common that they can express the influence of the oscillating motion due to reversing speeds as an additional factor $a_{o s c_{n}}$ for the basic life rating equation (1), see equation (2). Often, periodically changing speeds occur with periodically changing loads at the same time. The influence of variable load on the lifetime, either during constant speed or varying speed, can be expressed by the modification factor $a_{o s c_{P}}$, see equation (2).

$$
L_{10}=a_{o s c_{n}} \cdot a_{o s c_{P}} \cdot\left(\frac{C}{P}\right)^{p} \cdot 10^{6} \text { oscillations }
$$

\subsection{Oscillating Speed}

In the following, available approaches to consider the influence of varying speeds on the basic life rating are presented.

\subsubsection{Harris 1}

Regarding oscillating motion due to periodically reversing speeds, Harris and Kotzalas (2007) provide a modification to supplement the standard ISO 281, by considering the influence on the lifetime calculation through adjusting the experienced dynamic equivalent load $P$ on the bearing, see equation (3). The corresponding modification factor $a_{o s c_{n}}$, or oscillation factor, for this approach is given by equation (4). This approach is hereafter referred to as Harris 1.

$$
\begin{aligned}
P_{o s c} & =\left(\frac{2 \cdot \phi}{\pi}\right)^{\frac{1}{p}} \cdot P \\
a_{o s c_{n} H a 1} & =\frac{\pi}{2 \cdot \phi}
\end{aligned}
$$

With a decreasing oscillation amplitude $\phi$, the Harris 1 approach results in an increasing lifetime, see figure 3 .

This increase for smaller amplitudes is based on the consideration that only an increasingly smaller part of the circumference of the bearing is stressed and therefore the fatigue must be lower. The Harris 1 method is a commonly used approach in the industry due to its easy application. It can also be found in handbooks from bearing manufacturers, e.g. Schaeffler (2014).

\subsubsection{Harris 2}

Another similar approach, introduced by Rumbarger and Jones (1968) and taken up by Harris et al. (2009),

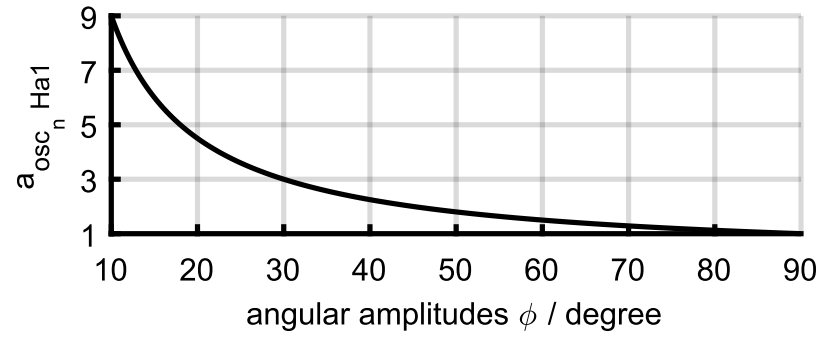

Figure 3: Oscillation modification factor of the Harris 1 approach, exemplary for angular amplitudes from $10^{\circ}$ to $90^{\circ}$

distinguishes two different cases regarding the stressed sections of the raceways. This approach is hereafter referred to as Harris 2. In the first case, in which the oscillation amplitude is larger than a specific critical angle $\phi_{\text {crit }}$, the sections of the raceways stressed by the individual rolling elements overlap, see figure 4 .
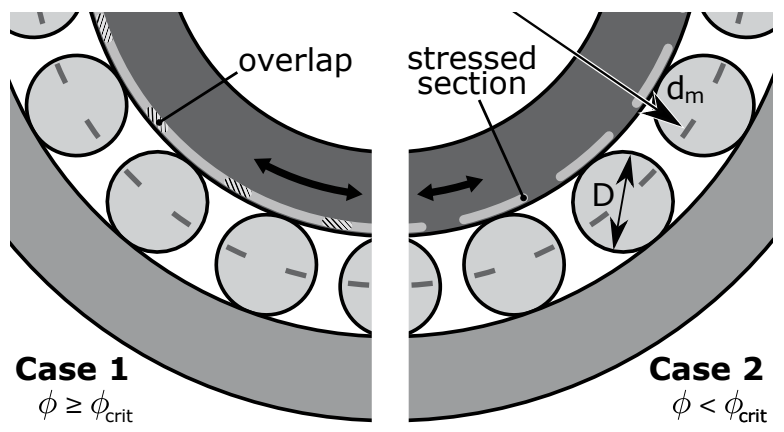

Figure 4: Case distinction of the Harris 2 approach, exemplary for inner race

The second case takes those oscillation amplitudes into account that are smaller than $\phi_{\text {crit }}$, meaning that the sections of the races stressed by the individual rolling elements border each other at most but do not overlap. The threshold angle $\phi_{c r i t}$ is given by equation (5), with $Z$ as the number of balls or rollers in the bearing and $\alpha$ as the contact angle. The parameters $D$ and $d_{m}$ are defined in figure 4 . The upper sign refers to the outer race, the lower sign to the inner race.

$$
\begin{aligned}
\phi_{c r i t} & =\frac{2 \cdot \pi}{Z \cdot(1 \mp \gamma)} \\
\gamma & =D \cdot \cos (\alpha) / d_{m}
\end{aligned}
$$

For the first case, the modification is performed by rating the bearing capacity $C$, see equation (6), similar to the first approach, given in equation (3). Transferred to an oscillation factor (equation (7)), it yields the same result as the Harris 1 method, see equation (4). 


$$
\begin{array}{r}
C_{o s c}=\left(\frac{\pi}{2 \cdot \phi}\right)^{\frac{1}{p}} \cdot C \\
a_{o s c_{n} H a 2}=a_{o s c_{n} H a 1}=\frac{\pi}{2 \cdot \phi}
\end{array}
$$

For the second case, an additional distinction is introduced between ball bearings and roller bearings. The modification for small oscillation angles adapts the bearing capacity as well and can similarly be transferred to modification factors. The modifications for ball bearings are given in equation (8) and (9).

$$
\begin{aligned}
C_{o s c} & =\left(\frac{\pi}{2 \cdot \phi}\right)^{\frac{3}{10}} \cdot Z^{0.033} \cdot C \\
a_{o s c_{n} H a 2} & =\left(\left(\frac{\pi}{2 \cdot \phi}\right)^{\frac{3}{10}} \cdot Z^{0.033}\right)^{p}
\end{aligned}
$$

For roller bearings, the modifications are similar to the ball bearings, but with different exponents, and are given in equation (10) and (11).

$$
\begin{aligned}
C_{o s c} & =\left(\frac{\pi}{2 \cdot \phi}\right)^{\frac{2}{9}} \cdot Z^{0.028} \cdot C \\
a_{o s c_{n} H a 2} & =\left(\left(\frac{\pi}{2 \cdot \phi}\right)^{\frac{2}{9}} \cdot Z^{0.028}\right)^{p}
\end{aligned}
$$

As a lower limit to the oscillation amplitudes for case 2, Harris et al. (2009) introduced the dither angle $\phi_{\text {dith }}$, see equation (12). The parameter $b$ refers to the semiminor axis of the contact ellipse in the contact between rolling element and raceway. The upper sign refers to the outer race, the lower sign to the inner race.

$$
\phi_{d i t h}=\frac{2 \cdot b}{d_{m}(1 \mp \gamma)}
$$

For oscillation amplitudes below this angle, fretting corrosion is most likely to occur and fatigue might not be the critical damage mechanism (Harris et al., 2009).

\subsubsection{Houpert}

Houpert (1999) provides another method, aiming to take the size $\epsilon$ of the load zone into account, see figure 5 .

The load zone size mainly depends on the load direction, the applied load, and the bearing clearance (Harris and Kotzalas, 2007). The parameter $\epsilon$ can be calculated using equation (13) and depends on the displacement (due to load or clearance) in axial $\left(\delta_{a}\right)$ and

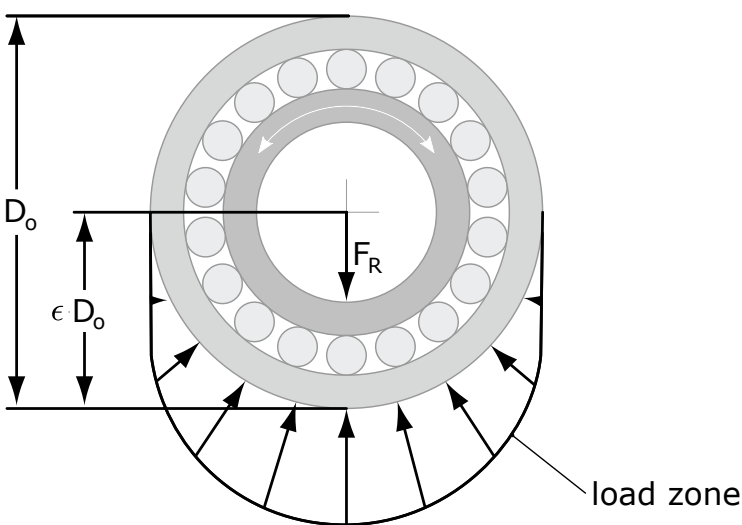

Figure 5: Load zone, exemplary for $\epsilon=0.5$

radial $\left(\delta_{r}\right)$ direction and the previously mentioned contact angle $\alpha$.

$$
\epsilon=\frac{1}{2} \cdot\left(1+\frac{\delta_{a} \cdot \tan \alpha}{\delta_{r}}\right)
$$

The advancement of this approach is the consideration of the length of the stressed section of the races in relation to the travelled arc for different oscillation angles. This approach is hereafter referred to as Houpert. The oscillation factor $a_{o s c_{n}}$ is calculated separately for inner and outer race and requires elaborate integral calculus. For more detailed information, it is referred to Houpert (1999). As a limitation of the described model, oscillation amplitudes $\phi \leq 2 \cdot \pi / Z$ are considered to cause failure due to wear and false brinelling rather than rolling fatigue. Exemplary for the inner race, the Houpert oscillation factor $a_{o s c_{n}}$ in comparison to the results of the Harris 1 method is given in figure 6 for an oscillation amplitude between $10^{\circ}-90^{\circ}$ and for a load zone size $\epsilon$ between $0.05-4$.

For larger load zone sizes, the results of the Houpert method are very similar to those from the Harris 1 method. The Houpert approach promises a higher calculation accuracy for load zone sizes $\epsilon<1$, compared to the Harris 1 approach (Harris and Kotzalas, 2007). For load zone sizes $\epsilon$ equal or greater than 1, the Harris 1 method yields sufficiently accurate results (Harris and Kotzalas, 2007). Since the pressures and the number of stress cycles are usually higher for the inner race than the outer race, the inner race is likely to fail earlier than the outer race. Therefore, taking only the oscillation factor for the inner race into account seems to be sufficient for the general life rating calculation (Houpert, 1999). 


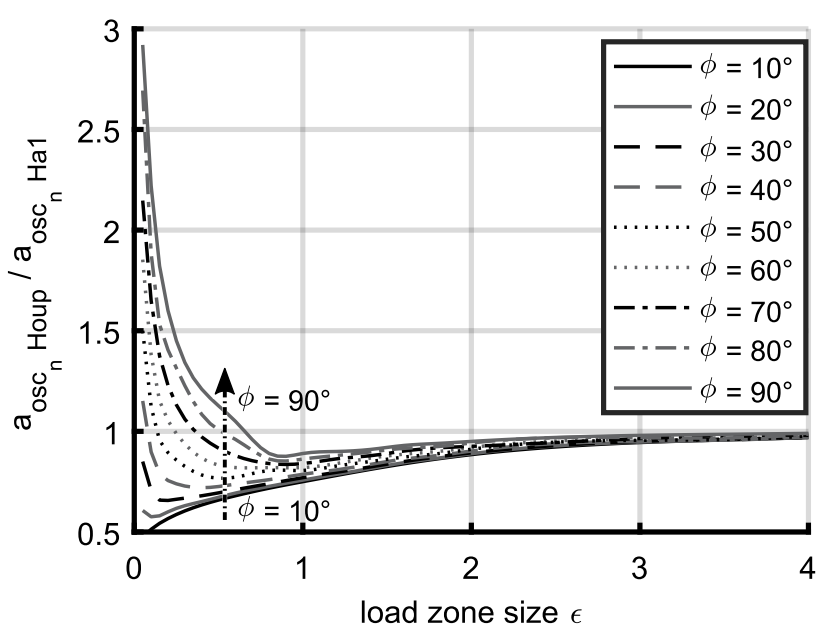

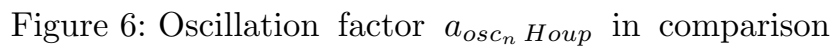
to the Harris 1 approach, according to (Houpert, 1999)

\subsection{Oscillating Load}

In many applications, oscillating loads can occur simultaneously to oscillating motion of the bearing. The influence of oscillating loads on the bearing lifetime during constant rotational speeds has been described by Harris and Kotzalas (2007). In that approach, an equivalent load $P_{e q}$ is determined to take the load variations into account with the basic lifetime equation (1). This equivalent load can be described in relation to the mean value $P_{\text {mean }}$ of the load and a load modification factor $\psi_{m}$, see equation (14).

$$
P_{e q}=\psi_{m} \cdot P_{\text {mean }}
$$

The load modification factor $\psi_{m}$ depends on the ratio of a steady load $P_{s}$ and an amplitude $P_{d}$ of a dynamic load, hereby described as the factor $\beta$, given by equation (15).

$$
\beta=\frac{P_{s}}{P_{s}+P_{d}}
$$

The parameter $\beta$ is illustrated in figure 7 for three exemplary load ratios. Presupposing an overall positive load, the lower limit of $\beta$ is 0.5 . For a load without a dynamic share, $\beta$ is 1 .

By applying the general formula from (Grote and Feldhusen, 2011), the load modification factor $\psi_{m}$ can be calculated by integrating the periodic load and speed over its period duration and dividing it by the steady load $P_{s}$, see equation (16). If speed and load oscillation are not in phase, the phase shift $\Delta \phi$ is unequal to zero and has to be considered.

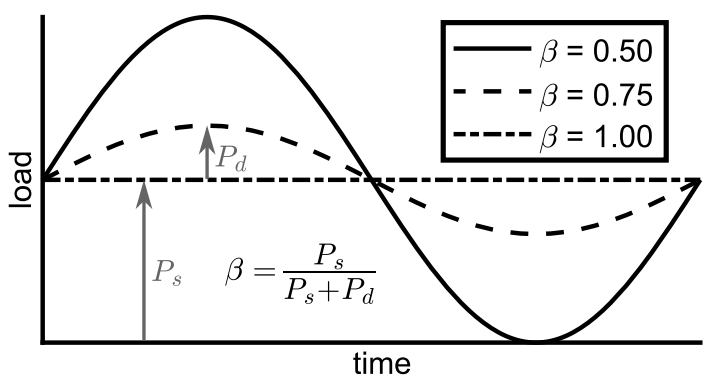

Figure 7: Load type

$$
\psi_{m}=\frac{\left\{\frac{\int_{0}^{2 \pi}\left\{P_{s}+P_{d} \cdot \sin (\phi+\Delta \phi)\right\}^{p} \cdot|\sin \phi| d \phi}{\int_{0}^{2 \pi}|\sin \phi| d \phi}\right\}^{1 / p}}{P_{s}}
$$

The influence of oscillating loads on the lifetime calculation can be expressed by the lifetime modification factor $a_{o s c_{P}}$, see equation (17). For this, the factor $\psi_{m}$ is transferred, using equation (18).

$$
\begin{aligned}
& L_{P_{o s c}}=a_{o s c_{P}} \cdot L_{P_{\text {const }}} \\
& a_{o s c_{P}}=\psi_{m}^{-p}
\end{aligned}
$$

The lifetime modification factor $a_{o s c_{P}}$, transferred from load modification factor $\psi_{m}$ for simultaneously oscillating loads and speeds, is given in figure 8 for three different exponents $p$ and with and without phase shift between speed and load oscillation.

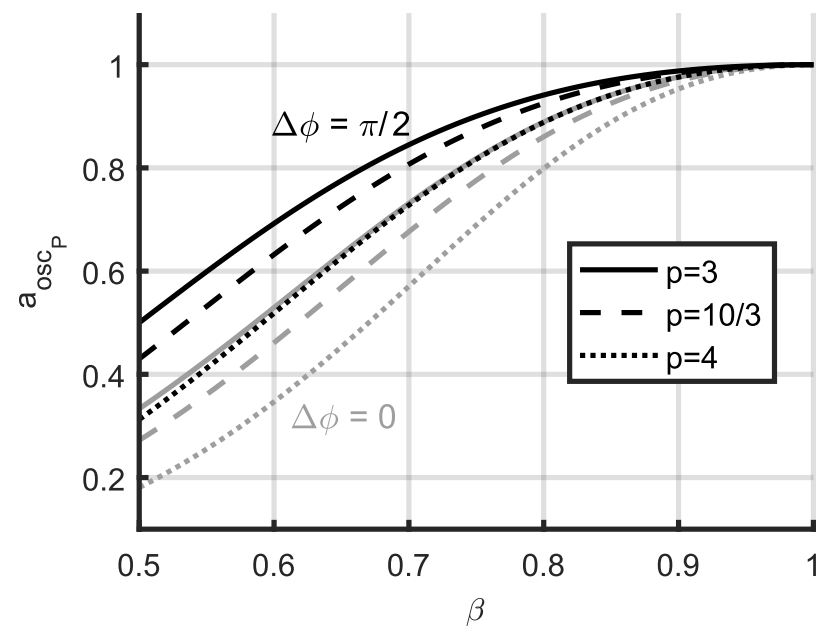

Figure 8: Lifetime modification factor $a_{o s c_{P}}$ for sinusoidal loads at sinusoidal speeds with and without phase shift

It can be observed, that oscillating loads, with and without phase shift to oscillating speeds, have a significant influence on the lifetime and can lead to calculated 
lifetimes of only $20 \%$ of the values calculated with only the mean value of the periodic load.

With both the influence of oscillating speed and oscillating load transferred into separate lifetime modification factors $a_{o s c_{n}}$ and $a_{o s c_{P}}$, the bearing lifetime influenced by reversing speeds and variable loads can be calculated using equation (2). The modification factors can be combined into an overall lifetime modification factor $a_{o s c}$, see equation (19).

$$
a_{o s c}=a_{o s c_{n}} \cdot a_{o s c_{P}}
$$

\subsection{Life Modification Factor $a_{I S O}$}

The basic lifetime rating, given by equation (1), is only valid for a sufficient lubrication film thickness and sufficiently clean lubricant without large particles. Since the lifetime of a rolling bearing is directly influenced by the lubrication regime, an additional modification in the form of the life modification factor $a_{I S O}$ has been introduced in the standard ISO 281, for deviating conditions. The calculation of this factor takes the influence of the lubrication regime on the lifetime rating into account. Including the factor $a_{I S O}$, the calculation of the modified rating life $L_{10 m}$ is given by equation (20). The previously described lifetime modification factor $a_{\text {osc }}$ can be applied as well, yielding the modified lifetime for oscillating bearings $L_{10 m_{\text {osc }}}$, see equation (21).

$$
\begin{aligned}
L_{10 m} & =a_{I S O} \cdot L_{10} \\
L_{10 m_{\text {osc }}} & =a_{I S O} \cdot a_{\text {osc }} \cdot L_{10}
\end{aligned}
$$

The available approaches for lifetime calculation of oscillating bearings only consider the effect of varying speed and load on the basic life rating calculation (equation (1)) and not the influence on the modified rating life calculation (equation (20)), expressed by the factor $a_{I S O}$. However, Houpert (1999) recommends using the mean speed as an estimation for the equivalent speed $n_{e q}$ as input for the calculation of the factor $a_{I S O}$, see equation (22).

$$
n_{e q}=f_{o s c} \cdot \frac{4 \cdot \phi}{2 \pi}=f_{o s c} \cdot \frac{2 \cdot \phi}{\pi}
$$

To yield the equivalent speed, the oscillation frequency is multiplied by the share of the travelled rotation, calculated by dividing the travelled angle per oscillation (back and forth is equivalent to $4 \cdot \phi$ ) with the angle of a whole rotation. This approach, however, cannot take the non-linear relation between speed, load, lubrication regime, and lifetime, reflected by the factor $a_{I S O}$, entirely into account.

\subsection{Conclusion}

In this section, different lifetime modification factors $a_{o s c_{n}}$ and $a_{o s c_{P}}$ have been described. The factors presented in subsection 2.1 and 2.2 aim to modify the standardized basic lifetime calculation for continuously rotating bearings to enable the calculation of the lifetime of bearings underlying oscillatory speeds and loads. Since the approaches are designed for sinusoidal speeds and loads, they cannot be applied to irregular nonsinusoidal speeds and loads in a simple way. The life modification factor $a_{I S O}$, presented in subsection 2.3, takes the influence of lubrication for continuously rotating bearings into account. For oscillating applications, the available approaches assume mean values for speed and load throughout a duty cycle for calculating the influence of lubrication on the lifetime. In the following, a numerical approach is discussed to address the mentioned shortcomings of existing approaches for stochastically oscillating motion and variable load behaviour.

\section{Numerical Approach}

The presented available approaches, analysing load and speed oscillations described in section 2, can only consider sinusoidal speed and sinusoidal load oscillations for fatigue lifetime calculations using the standard ISO 281. This means, that whenever an oscillating motion is irregular or stochastic, the speed has to be approximated by a sine, either for the entire duty cycle or per oscillation cycle. The same applies to varying loads. Depending on the application, this could mean a significant loss of information and thus a significant error in lifetime calculation.

To address the mentioned difficulties to match irregular oscillations to the available models, another approach is used, which analyses the speed and load incrementally without superordinate detection of oscillations. The approach consists of dividing the load and speed history into finite time steps to which the modified life rating calculation given by equation (20) is applied individually. Since each time step yields a different travelled angle depending on the present load, the approach consists of adding up damage, caused by present speed and load during a finite small angle and rating it by the ratio of travelled angle to full rotation.

In the following, the described approach is demonstrated. In figure 9, an exemplary section of an irregular data set consisting of the rotational speed $n$ of a bearing, the dynamical equivalent load $P$, and the rotation angle is illustrated. An exemplary section with a duration of $3.5 \mathrm{~s}$ is shown. The data points are available with a resolution of $0.1 \mathrm{~s}$. 


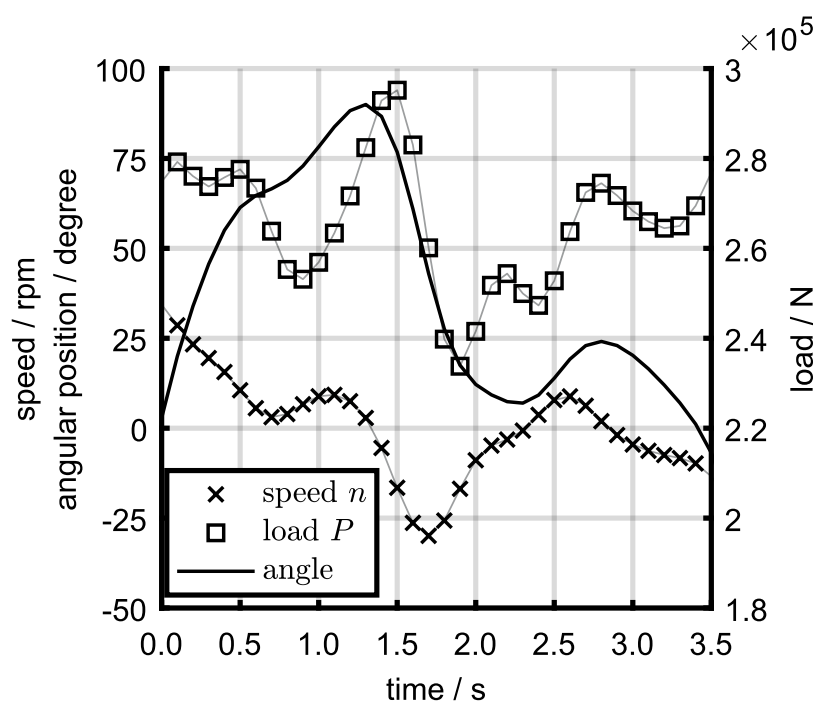

Figure 9: Data set for illustration of numerical approach

It can be observed, that the bearing is in an oscillating condition due to a reversing rotational speed. At the same time, the load is subject to fluctuation as well. Both, load and speed, are non-sinusoidal and therefore not a suitable input for the available approaches aiming to calculate the lifetime of oscillating bearings. Using the given exemplary data set, a numerical approach for stochastic speeds and loads is presented.

First, for each data point $i$, the damage on the bearing is calculated by equation (20), using the present load $P_{i}$, speed $n_{i}$ and travelled distance $\Delta \phi_{i}$ (product of time step and present speed). The ratio of the travelled distance $\Delta \phi_{i}$ to a full rotation is used to calculate the amount of damage caused by the conditions defined by each data point, according to the linear damage accumulation theory by Palmgren. The factor $a_{I S O}$, representing the lubrication condition, is calculated for each data point using the present load and present speed. This damage calculation is repeated for each data point, and eventually the overall damage $D_{\text {sequence }}$, caused by the load and speed sequence, can be determined, see equation (23). Eventually, equation (24) yields the lifetime $B_{10}$ in hours by extrapolating the duration $T_{\text {sequence }}$ of the analysed sequence by the determined damage $D_{\text {sequence }}$.

$$
\begin{aligned}
D_{\text {sequence }} & =\sum_{i=1}^{n} \frac{\frac{\Delta \phi_{i}}{2 \cdot \pi}}{a_{I S O_{i}} \cdot\left(\frac{C}{P_{i}}\right)^{p} \cdot 10^{6}} \\
B_{10} & =\frac{T_{\text {sequence }}}{D_{\text {sequence }}}
\end{aligned}
$$

The advantage of this approach is firstly the possibility of calculating the basic life rating of oscillating bear- ings with non-sinusoidal loads and speeds and secondly, the consideration of varying speeds and loads for the life modification factor $a_{I S O}$, necessary for the modified life rating calculation in the ISO 281 standard. The numerical approach lacks the capability of taking sophisticated distinctions into account, as Harris 2 does with the critical angle distinction and Houpert does with comparing the oscillation amplitude to the load zone size.

\section{Comparison of calculation approaches using artificial duty cycles}

In the following, the approaches that have been presented in section 2 and 3, are compared for exemplary artificial duty cycles, consisting of sinusoidal and constant speeds and loads. The duty cycles cause an oscillation with an amplitude of $\phi=10^{\circ}$ around the centre position, see figure 2. The load oscillations have the same period duration as the speed oscillations and the ratio of the mean load value and amplitude height is given by $\beta$ in table 1 .

\begin{tabular}{|c|c|c|}
\hline $\begin{array}{l}\text { duty } \\
\text { cycle }\end{array}$ & $\begin{array}{c}\text { speed } \\
n\end{array}$ & $\begin{array}{l}\text { ad } \\
P\end{array}$ \\
\hline 1 & & $\beta=1$ \\
\hline 2 & & $\begin{array}{c}\beta=0.75 \\
\Delta \phi=0\end{array}$ \\
\hline 3 & & $\begin{array}{c}\beta=0.75 \\
\Delta \phi=\pi / 2\end{array}$ \\
\hline
\end{tabular}

Table 1: Parameters of artificial duty cycles

For the exemplary bearing specimen, a radial cylindrical roller bearing with a contact angle of $\alpha=0^{\circ}$, and $Z=22$ rolling elements is selected. For the Harris 2 approach, a critical amplitude angle of $\phi_{c r i t}=15^{\circ}$ for the inner race and $\phi_{\text {crit }}=18^{\circ}$ for the outer race can be determined, using equation (5). The selected oscillation amplitude of $\phi=10^{\circ}$ is below the critical angle, therefore the rollers' overrun sections are not overlapping (Harris 2: Case 2). A life exponent $p=10 / 3$ is chosen, along with a load zone size $\epsilon=0.5$ (maximum value for radial rolling bearings under purely radial load, see figure 5), required for Houpert's method.

Since the bearing capacity $C$ and the mean dynamical equivalent load $P_{\text {mean }}$ are the same for all duty cycles, the results will be given as lifetime modifica- 
tion factors considering the influence of speed $a_{o s c_{n}}$ and load $a_{\text {osc }_{P}}$ for comparison, rather than lifetimes. The results for the first duty cycle with sinusoidal speed and constant load are given in figure 10.

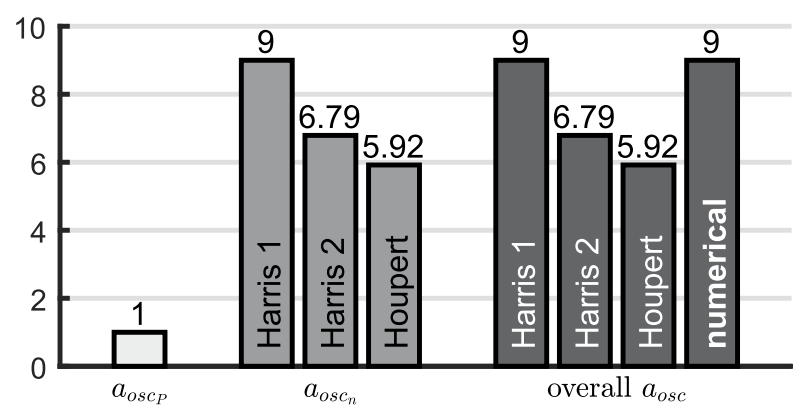

Figure 10: Lifetime modification factors for the first artificial duty cycle

For the first duty cycle, the lifetime modification factors $a_{o s c_{n}}$ take values between 5.92 and 9. The modification factors $a_{o s c_{n} H a 1}$ and $a_{o s c_{n} \mathrm{Ha} 2}$ are unequal, because the oscillation amplitude is below the critical angles $\phi_{\text {crit }}$, causing the Harris 2 method to yield a lower lifetime, compared to the Harris 1 result. The result calculated using the Houpert method is lower than the results of Harris 1 and Harris 2. For the given oscillation amplitude of $\phi=10^{\circ}$, the Houpert result will decrease even further, for a load zone size lower than the assumed $\epsilon=0.5$. For the first duty cycle, the lifetime modification factor due to load is $a_{o s c_{P}}=1$, because the load is constant and therefore the equivalent load is equal to the mean load value, see figure 8 . Thus, the overall modification factors $a_{o s c}$, including the load modification and the speed modifications by Harris 1, Harris 2 and Houpert are equal to the speed modification factors $a_{o s c_{n}}$. The numerical approach yields the same result as the overall oscillation factor, calculated using the Harris 1 approach.

The second duty cycle differs from the first by introducing an oscillating load, with a load ratio factor $\beta$ of 0.75 with phase equivalence to the speed oscillation. The calculation results of the lifetime modification factors for this duty cycle are given in figure 11 .

Since the speed behaviour is identical to the speed condition in the first duty cycle, the lifetime modification factors $a_{o s c_{n}}$ are the same as the results in figure 10. With the oscillating load, in phase with the speed oscillation, the lifetime modification factor $a_{o s c_{P}}$

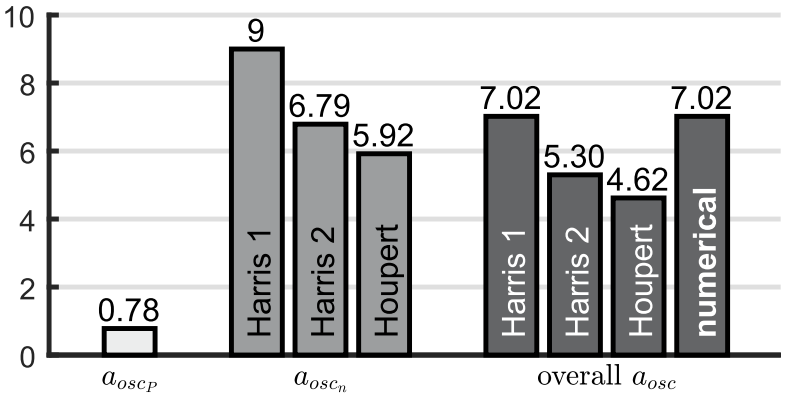

Figure 11: Lifetime modification factors for the second artificial duty cycle

due to load is 0.78 . Thus, the overall lifetime modification factors $a_{\text {osc }}$ are lower than the results of the first investigated duty cycle. Again, the numerical approach yields the same result as the overall oscillation factor calculated using the Harris 1 modification.

In duty cycle 3 , a phase shift of $\Delta \phi=\pi / 2$ between speed and load oscillation is introduced. This altered condition has an influence on the calculation of the lifetime modification factor $a_{o s c_{P}}$, which is now 0.87 , see figure 12.

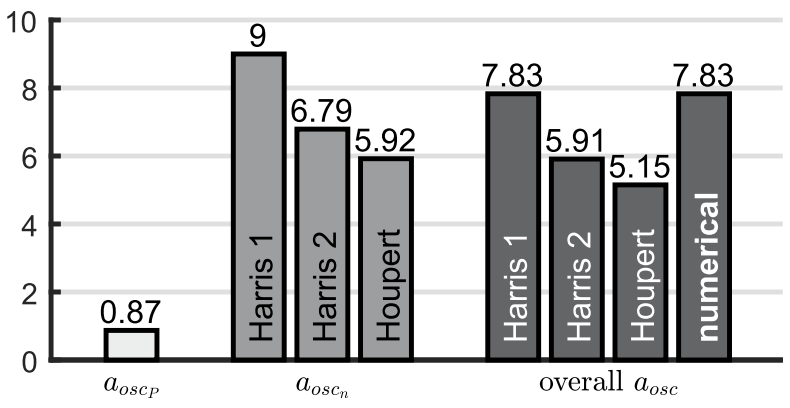

Figure 12: Lifetime modification factors for the third artificial duty cycle

The overall lifetime modification factor $a_{\text {osc }}$, calculated by the Harris 1 approach and the factor calculated by the numerical approach are again equal.

As a conclusion it can be stated, that the numerical approach described in section 3 yields the same results as the equation $a_{o s c H a 1}=a_{o s c_{n} H a 1} \cdot a_{o s c_{P}}$ for each duty cycle. The numerical approach can therefore be considered as an equivalent to the Harris 1 method for sinusoidal oscillation behaviour for the calculation of the basic life rating, see equation (1).

Table 2: Parameters for comparison with artificial duty cycles

\begin{tabular}{c|c|c|c|c|c|c|c|c|c|c|c}
$\beta$ & $Z$ & $\phi_{\text {crit }_{\text {inner }}}$ & $\phi_{\text {crit }_{\text {outer }}}$ & $\phi$ & $p$ & $\epsilon$ & $\gamma$ & $f_{\text {osc }}$ & Temp & $\nu_{40}$ & $e_{c}$ \\
\hline 0.75 & 22 & $15^{\circ}$ & $18^{\circ}$ & $10^{\circ}$ & $10 / 3$ & 0.5 & 0.108 & $1.65 \mathrm{~Hz}$ & $40^{\circ} \mathrm{C}$ & $460 \mathrm{~mm}^{2} / \mathrm{s}$ & 0.55
\end{tabular}


For the calculation of the life modification factor $a_{I S O}$, information about bearing speed, load and lubrication is required, among other parameters. For comparison to the numerical calculation, an equivalent factor $a_{I S O \text { mean }}$, for the entire duty cycle, is calculated, using the mean load value and a mean speed, corresponding to equation (22). The factor $a_{I S O \text { num }}$ from the numerical method is calculated from the individual factors $a_{I S O_{i}}$ for the finite time steps of the duty cycle, compare to equation (24). The necessary parameters for calculation of the factor $a_{I S O}$ according to ISO 281 (2007) can be found in table 2.

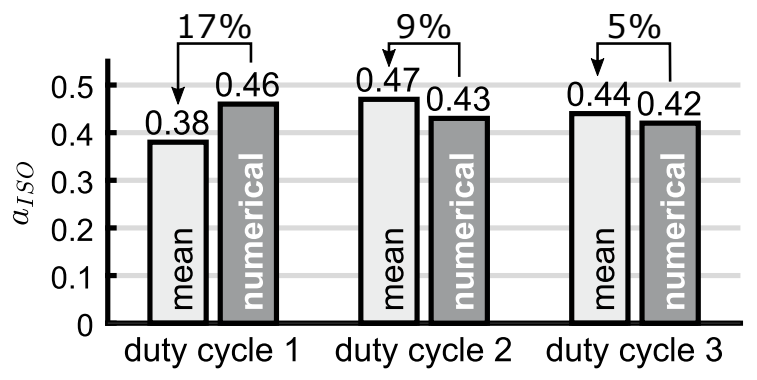

Figure 13: ISO 281 life modification factor $a_{I S O}$ for the three artificial duty cycles

The results are given in figure 13. For the first duty cycle, with sinusoidal speed and constant load, the calculation result of the factor $a_{I S O}$, using the mean values per oscillation cycle, differs from the factor using the numerical approach by approximately $17 \%$. For the other two load cases with sinusoidal loads, the results differ from the numerical results by approximately $9 \%$ for a phase shift $\Delta \phi=0$ and by $5 \%$ for a phase shift $\Delta \phi=\pi / 2$. In this example the approach using the mean speed and mean load for calculating the factor $a_{I S O}$ yields different results than the numerical method. However, since the $a_{I S O}$ factor depends on numerous parameters, a universal statement about the influence of the calculation approach cannot be made and only serves as an example in this work, showing that there can be a significant difference between the numerical calculation and the available approaches using mean values for bearing speed and load.

\section{Results for Offshore Crane Winch Drivetrain}

To compare the previously described calculation approaches and to demonstrate the influence of irregular, non-sinusoidal bearing speeds and loads on the lifetime calculation, the oscillating motion of an active heave compensated (AHC) offshore crane winch is investigated. This AHC mode enables the crane operator to maintain a steady vertical position of the payload, even when the ship moves due to the wave motion. Since the waves induce irregular but periodical motion on the ship and subsequently on the crane and the payload, the drives need to coil and uncoil the winch drum anticyclically to the wave motion to compensate the vertical payload movement, see figure 14.

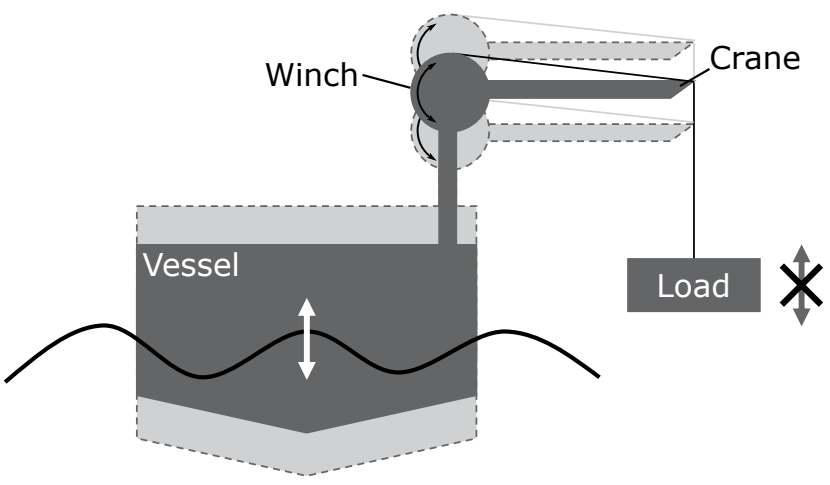

Figure 14: Oscillation due to Active Heave Compensation (AHC)

The active heave compensation causes oscillating speeds and oscillating loads (see figure 15) due to acceleration and deceleration of the rotational inertia of the drivetrain and winch drum. To obtain the load and speed data, either measurement data can be used, or as in this case, the required information can be provided using a wave prediction model. The used JONSWAP model (Hasselmann et al., 1973) enables the calculation of the time history of the vertical wave position, velocity and acceleration, from which the rotating angle, rotational speed and rotational acceleration of the winch can be determined. For more detailed information about the system and its loads, it is referred to Wöll et al. (2017a,b,c).

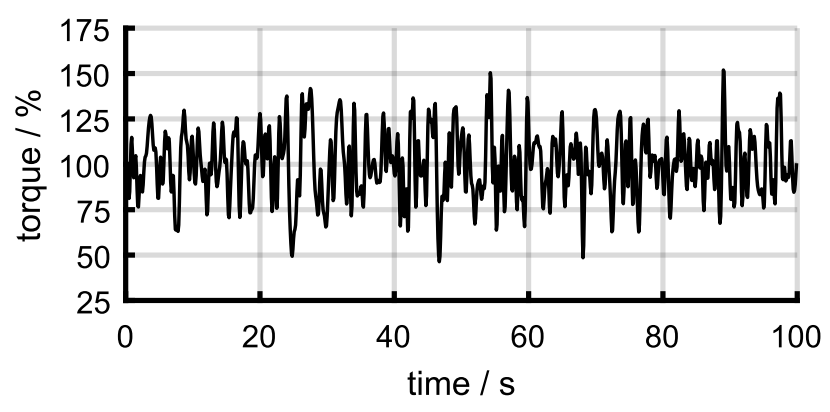

Figure 15: Section of torque history at gearbox output

For the investigation carried out in this paper, the information listed in table 3 is used for the JONSWAP wave model. The significant wave height $H_{s}$ and the average wave period $T_{1}$ correspond to a wave motion 
on the North Sea during a wind speed of approximately $10 \mathrm{~m} / \mathrm{s}$ (Journée and Massie, 2001).

Table 3: Parameters for JONSWAP model \begin{tabular}{l|l} 
Significant wave height $H_{s}$ & $1.65 \mathrm{~m}$
\end{tabular}

\begin{tabular}{l|l}
\hline Wave period $T_{1}$ & $5.1 \mathrm{~s}$ \\
\hline Carried payload & $75 \mathrm{t}$ \\
\hline Operating depth & $200 \mathrm{~m}$ \\
\hline Peakedness factor & 3.3
\end{tabular}

The loads on the bearings are determined by building transfer functions to connect bearing loads with gearbox torque, based on the gearbox dimensions and bearing positions. The rotational speeds and angular positions of each bearing are determined by transforming the gearbox output speed and angle based on the gear stage ratios. Similar to section 4, the different calculation approaches for oscillating bearings are applied to the bearings. However, the calculations for the Harris 1, Harris 2 and Houpert approaches have to be modified, by transferring the non-sinusoidal load and speed signal to a compatible data set. First, the time points for each bearing are identified, at which the rotation passes the bearing centre position. By determining the maximum angle in between the centre positions, the oscillation amplitudes are found and the mean value of the two adjacent oscillation amplitudes per centre crossing is assigned to a half-oscillation. In a next step, for each half-oscillation (means rotation from one turning point to the next) the mean speed and mean load are determined.

Now, the theoretical lifetime for the conditions of each half-oscillation can be calculated using the lifetime equation and the different life modification factors. The load zone size $\epsilon$ for the Houpert calculation is set to 0.5 for each bearing, which is the maximum value for radial rolling bearings under purely radial load, see figure 5 . The critical angle $\phi_{\text {crit }}$ as threshold for the calculation of $a_{o s c_{n} \mathrm{Ha} 2}$ is calculated individually for each bearing, using equation (5) for the inner race. For the numerical method, these considerations are not necessary, since the data set is investigated per data point and not per half-oscillation.

Utilizing the system reliability evaluation method presented in Neumann et al. (2016), all bearings are assigned with failure distributions, depending on their lifetimes. Subsequently, based on the failure probabilities and the system structure, the overall system failure distribution can be determined, from which the system lifetime, which is mainly determined by bearing failures, can be derived.
Since the system failure probability and the system lifetime are influenced by the lifetime and number of the components, it is not purposeful to perform the quantitative comparison of the models on a system level. Therefore the most critical bearing having the lowest lifetime, thus the most critical, is selected and the lifetimes are compared. The lifetime calculation results for the most critical bearing, which was identified using the reliability evaluation, are listed for comparison in table 4 and are normalized to the result of the numerical approach.

Table 4: Critical bearing lifetime comparison

\begin{tabular}{c|cccc} 
& Numerical & Harris 1 & Harris 2 & Houpert \\
\hline$B_{10}$ & $100 \%$ & $109 \%$ & $105 \%$ & $137 \%$ \\
\hline$a_{I S O}$ & 0.90 & 0.99 & 0.99 & 0.95
\end{tabular}

Evidently, the numerical approach yields the highest failure probability and thus the lowest lifetime. The Houpert method yields the highest lifetime. The overall lifetimes calculated with the Harris 1 and Harris 2 methods are similar, due to the fact that only $2 \%$ of the half oscillation amplitudes are below the critical angle $\phi_{\text {crit }}$. The Houpert method yields a lifetime, which is $37 \%$ higher than the numerical result. Separately listed in table 4 are the calculated life modification factors $a_{I S O}$ for each approach. For this duty cycle the overall $a_{I S O}$ factors deviate by about $10 \%$ from the numerical result.

\subsection{Conclusion}

Evidently, the lifetime calculation results significantly depend on the used approach. The deviation of the Houpert result can be explained by the capability of the Houpert method to consider the assumed load zone size of $\epsilon=0.5$. The difference between the Harris 1 method and the numerical approach is solely due to the lack of accuracy by using mean speed and load values per half-oscillations, compared to the numerical approach. Presumably, the numerical approach provides more accurate results in comparison to the Harris 1 method for irregular oscillations.

As the numerical approach and the Harris 1 approach are equivalent for sinusoidal bearings speeds and loads, the statement can be made, that the available approaches designed for sinusoidal speeds and loads, overestimate the lifetime of irregular bearing speeds and loads due to the required approximations. For the given example, the difference between the calculated lifetime of the Harris 1 and the numerical approach is about $9 \%$. It must be assumed that Harris 2 
and Houpert overestimate the lifetime as well, due to the similar consideration principle for sinusoidal oscillations.

Furthermore, lifetime calculation with the presented approaches, based on the standard ISO 281, are not capable of distinguishing between oscillations around one single centre position or centre positions statistically uniformly distributed around the circumference. However, previous investigations consider only one single centre position, whereas for the analysed offshore crane winch a distribution of centre positions is most likely.

\section{Summary}

In this work, the available calculation approaches for the determination of the lifetime of oscillating bearings have been presented and discussed. A modified numerical approach has been described, which analyses occurring loads and speeds on the bearings during oscillating behaviour for finite small time steps instead of whole oscillations. This approach was subsequently compared to the literature approaches using artificial sinusoidal duty cycles, as well as irregularly oscillating bearing loads and speeds occurring in an offshore crane winch drivetrain. The results of the oscillation factors for speed and load, as well as the lifetime modification factor $a_{I S O}$, calculated by both a simplified and a numerical approach have been presented.

For the basic life rating calculation for oscillating bearings underlying sinusoidal speeds and loads, the numerical approach yields the same results as the most common available Harris 1 approach and can therefore be considered equivalent. The numerical approach lacks the sophisticated considerations in the Harris 2 approach (distinction between small and large oscillation amplitudes) and the Houpert approach (comparing oscillation amplitude and length of stressed race arc), leading to a deviation to the results of these approaches.

The separate analysis of the calculation of the factor $a_{I S O}$ for the modified lifetime calculation, results in mentionable differences between the simplified approaches and the numerical approach, for both, regular and irregular oscillations. The simplified calculation based on mean speed and mean load per half oscillation results in higher values, thus underestimating the caused damage, in comparison to the numerical method.

For the example of irregularly oscillating bearings due to non-sinusoidal speeds and loads, a significant difference of $9 \%$ between the lifetimes calculated by the Harris 1 approach, using averaged speed and load values, and the presented numerical approach can be observed. Considering that these approaches are equivalent for sinusoidal speeds and loads, the difference in the lifetime calculation leads to the assumption that the Harris 1 approach overestimates the lifetime of bearings underlying irregularly oscillating loads and speeds. Due to the similarity of the Harris 2 and Houpert approach to the Harris 1 method, it can be assumed that the available approaches overestimate the lifetime for non-sinusoidal loads and speeds.

All methods, including the proposed numerical approach, are not able to distinguish between a bearing oscillation around a single centre position or a statistically uniform distribution of the centre position around the circumference during its service life. This is due to the incapability of the calculations used in the standard ISO 281 to distinguish such cases. In future, the advanced considerations of the Harris 2 and Houpert approach need to be applied to the presented numerical approach as well. Furthermore, the difference of oscillations around the same centre position and uniformly distributed oscillation centre positions need to be investigated more thoroughly.

\section{Acknowledgements}

The research presented in this paper has received funding from the Norwegian Research Council, SFI Offshore Mechatronics, project number 237896.

\section{References}

Grote, K.-H. and Feldhusen, J. Dubbel. Springer, Berlin, Heidelberg, 2011. doi:10.1007/978-3-64217306-6.

Harris, T. A. and Kotzalas, M. N. Essential Concepts of Bearing Technology, volume 1 of Rolling bearing analysis. Taylor \& Francis, Boca Raton, 5. ed. edition, 2007.

Harris, T. A., Rumbarger, J. H., and Butterfield, C. P. Wind Turbine Design Guideline DG03: Yaw and Pitch Rolling Bearing Life. 2009. doi:10.2172/969722.

Hasselmann, K., Barnett, T. P., Bouws, E., Carlson, H., Cartwright, D. E., Enke, K., Ewing, J. A., Gienapp, H., Hasselmann, D. E., Kruseman, P., Meerburg, A., Müller, P., Olbers, D. J., Richter, K., Sell, W., and Walden, H. Measurements of wind-wave growth and swell decay during the Joint North Sea Wave Project (JONSWAP). Hamburg, 1973. 
Houpert, L. Bearing Life Calculation in Oscillatory Applications. Tribology Transactions, 1999. 42(1):136143. doi:10.1080/10402009908982200.

ISO 281. Rolling bearings - Dynamic load ratings and rating life. 2007.

Journée, J. M. J. and Massie, W. W. Offshore Hydromechanics. Delft University of Technology, first edition, 2001.

Lundberg, G. and Palmgren, A. Dynamic Capacity of Rolling Bearings, volume 1:3 of Acta polytechnica Mechanical engineering series. Stockholm, 1947.

Neumann, S., Wöll, L., Feldermann, A., Strassburger, F., and Jacobs, G. Modular System Modeling for Quantitative Reliability Evaluation of Technical Systems. Modeling, Identification and Control, 2016. 37(1):19-29. doi:10.4173/mic.2016.1.2.

Rumbarger, J. H. and Jones, A. B. Dynamic Capacity of Oscillating Rolling Element Bearings. Journal of Lubrication Technology, 1968. 90(1):130. doi:10.1115/1.3601528.
Schaeffler. Rolling bearings: Catalogue HR 1. Schaeffler Technologies AG \& Co. KG, 2014.

Wöll, L., Feldermann, A., and Jacobs, G. Sensitivity Analysis on the Reliability of an Offshore Winch Regarding Selected Gearbox Parameters. Modeling, Identification and Control, 2017a. 38(2):51-58. doi:10.4173/mic.2017.2.1.

Wöll, L., Jacobs, G., Feldermann, A., Neumann, S., and Strassburger, F. Einfluss der Wellenhöhe auf die Zuverlässigkeit einer Offshore-Winde. In G. Jacobs, editor, Antriebstechnisches Kolloquium 2017, Antriebstechnisches Kolloquium. Books on Demand, Norderstedt, pages 59-70, 2017b.

Wöll, L., Schick, K., Jacobs, G., Kramer, A., and Neumann, S. Reliability Evaluation of Drivetrains: Challenges for Off-Highway Machines. In C. Volosencu, editor, System Reliability. InTech, 2017c. doi:10.5772/intechopen.70280. 